\title{
Common Host Factors Involved in the Function of the Foot-and-mouth Disease Virus and Classical Swine Fever Virus Internal Ribosomal Entry Sites
}

Yutaro Ide

Kagoshima University

Nobumasa Ito

Kagoshima University

Takafumi Matsui

Kagoshima University

Kyoko Tsukiyama-Kohara ( $\nabla$ kkohara@vet.kagoshima-u.ac.jp )

Kagoshima University https://orcid.org/0000-0002-4403-2018

\section{Research Article}

Keywords: Foot-and-mouth disease virus, classical swine fever virus, internal ribosomal entry site, Pycnogenol, PKD1L3, USP31, translation

Posted Date: March 23rd, 2021

DOl: https://doi.org/10.21203/rs.3.rs-327980/v1

License: (c) (i) This work is licensed under a Creative Commons Attribution 4.0 International License. Read Full License 


\section{Abstract}

Foot-and-mouth disease virus (FMDV) and classical swine fever virus (CSFV) both possess positive strand RNA genomes and an internal ribosomal entry site (IRES) element within their 5 c-untranslated regions. To identify common host factors involved with the activity of these IRESes, we utilized cell lines expressing a bicistronic luciferase reporter plasmid, which contained an FMDV-IRES or CSFV-IRES element between the Renilla and firefly luciferase genes. First, we treated FMDV-IRES cells with French maritime pine extract, Pycnogenol ${ }^{\circledR}$ (PYC), and evaluated its suppressive effect on FMDV-IRES activity, as anti-viral effect of PYC was reported so far. We next performed microarray analysis to identify host factors affected by PYC, and confirmed host-factor-IRES interaction by applying host factor-specific siRNAs. We found that polycystic kidney disease 1-like 3 (PKD1L3) and ubiquitin specific peptidase 31 (USP31) are involved in FMDV-IRES activity. Moreover, silencing of these factors also significantly suppressed CSFV-IRES activity. Accordingly, we suggest that PKD1L3 and USP31 are host factors that are involved in the function of the FMDV and CSFV-IRES elements.

\section{Introduction}

Foot-and-mouth disease (FMD) and classical swine fever (CSF) are highly contagious viral diseases that affect cloven-hoofed animals [8] and swine[21], respectively. The causative pathogens for these diseases - FMD virus (FMDV; genus Aphthovirus, family Picornaviridae) and CSF virus (CSFV; genus Pestivirus, family Flaviviridae) - possess positive-sense, single-stranded RNA genomes. For both of these genomes, translation of the virus-encoded polyprotein is directed by an internal ribosomal entry sites (IRES) within a relatively long 5'-untranlated region (5'UTR). The FMDV-IRES is classified as a type II IRES, a type which also exists in the cardioviruses (e.g. encephalomyocarditis virus) 5'UTR [1]. CSFV-IRES is classified as a hepatitis $C$ virus (HCV)-like IRES [20, 22]. In an HCV-like IRES, domains II-IV are required for IRES activity, and HCV-like IRESes are shorter and more compact than type I and II picornavirus IRESes [11]. Common host factors (other than canonical translation initiation factors) essential in these IRES activities have not been fully characterized to date $[5,13]$ except proteomic analysis of interacting proteins $[18,19]$.

French maritime pine extract, Pycnogenol ${ }^{\circledR},(P Y C$; a registered trademark of Horphag Research, Geneva, Switzerland) is produced from the outer bark of the maritime mine tree (Pinus pinasterssp. Atlantica), and is considered to be a promising therapeutic agent with potential applications for human health (American Botanical Council, 2010). PYC inhibits encephalomyocarditis virus replication in the mouse heart by suppressing expression of proinflammatory cytokines [15], inhibit binding of human immunodeficiency virus type-1 to cells [3], and shows an inhibitory effect against replication for HCV in vitro and in vivo[2].

In this study, we aimed to elucidate host factors involved in both the FMDV-IRES and the CSFV-IRES, initial identification based on PYC treatment of IRES-expressing cells, and subsequent comprehensive analyses with the application of siRNAs. 


\section{Material And Methods}

\section{Cell culture and plasmids}

The human kidney cell line (HEK293) used in this study was obtained and cultured as previously described $[9,14]$.

The pRF vector containing an FMDV-IRES [serotype C; the 5'-UTR sequence (nucleotide numbers 5691,038 in FMDV serotype C, AF274010.1))] [12], was kindly donated by Dr. Hirasawa of the Memorial University of Newfoundland, and those containing an CSFV-IRES [4] were kind gift from Professor Graham J. Belsham of the University of Copenhagen. The pCAGGS-Neo vector was constructed using pCAG Neo (Fujifilm Wako, Tokyo, Japan) and pCAGGS vectors (cat. no. RDB08938; Riken Bank, Ibaraki, Japan). The CSFV-IRES cDNA (nt. 124 to 401) was excised from a reporter plasmid [4] using EcoRI and $\mathrm{NcOl}$, and inserted between the Renilla and firefly luciferase genes. Reporter genes were excised using the restriction endonucleases EcoRV (Toyobo, Osaka, Japan) and BamHI (New England Biolabs, Ipswich, MA, USA) and a pCAGGS-Neo/CSFV-IRES vector was generated by inserting a reporter gene into pCAGGS-Neo, which was then treated with EcoRV (Toyobo), BamHI (New England Biolabs), and rAPid alkaline phosphatase (Roche, Basel, Switzerland) using ligation mixture (Mighty Mix, Takara, Shiga, Japan).

The cells expressing the pCAGGS-Neo-CSFV-IRES were established, as described previously [14]. We also used cells expressing the pCAGGS-Neo-FMDV-IRES expressing cells (clone B5 and B10), which were established previously [14].

DNA sequencing was performed by FASMAC Co. (Kanagawa, Japan), and DNA sequence characterization was performed using GENETYX-Mac software (GENETYX Co., Tokyo, Japan) and GENBANK.

Cell viability was evaluated using WST assays (Dojindo, Kumamoto, Japan) by determining the optical density at $450 \mathrm{~nm}\left(\mathrm{OD}_{450}\right)$, according to the manufacturer's instructions. Luciferase assays were performed using a Dual-Luciferase Reporter Assay System (Promega, Madison, WI, USA). Luminescence was measured with a GloMax 96 Microplate Luminometer (Promega) for $10 \mathrm{~s}$, as previously described [9].

\section{RNA isolation and microarray analysis}

Total RNA was extracted using ISOGEN (NIPPON GENE Co. Tokyo, Japan) from PYC -treated $(10 \mu \mathrm{g} / \mathrm{mL}$ $72 \mathrm{hrs}$ ) and untreated B10 cells. We have chosen linear phase of PYC treatment. RNA quality was measured using an Agilent 2100 bioanalyzer and showed 9.8 RIN (maximum 10 and more than 7.0 is applicable for analysis). Microarray analysis was performed by Hokkaido System Science Co., Ltd. (Sapporo, Japan), using microarray slides (SurePrint G3 Human 8x60K ver 3.0) (Agilent Technologies Co., Santa Clara, CA). RNA samples were labeled with Cy3 or Cy5, hybridized with slides using a gene expression hybridization kit (Agilent Technologies Co.), washed with gene expression wash buffer (Agilent Technologies Co.) and scanned with a microarray scanner (G2505C, Agilent Technologies Co.). All data were calculated using Agilent feature extraction (12.0.3.1). 


\section{Short interfering RNA (siRNA) transfection}

We used siRNAs targeting polycystic kidney disease 1-like 3 (PKD1L3), ubiquitin specific peptidase 31 (USP31), and signal-regulatory protein gamma (SIRPG) which were designed using BLOCK-iT RNAi Designer (Thermo Fisher Scientific, Waltham, MA, USA). The sequences were: 5'CAGUUCAUGGUUUGCAAGCUCUUAA-3', 5'- CAGCACAGCCG-

CGACUUCAAGACUA-3' and5'- CGGCACAUACUACUGUGUGAAGUUU-3', respectively. For control siRNA, we used ON-target plus siRNA control (Horizon/Dharmacon, Lafayette, CO, USA). siRNA (5 nM) reverse transfection was performed using Lipofectamine RNAiMAX reagent (Invitrogen) according to the manufacturer's specifications.

\section{Statistical analysis}

All data are presented as means \pm standard deviations from three independent experiments. Statistical analysis was performed using Student's $t$-tests to evaluate significant differences. Results with $P$ values of less than 0.05 were considered significant.

\section{Results}

\section{Effect of Pycnogeno/® on FMVD-IRES activity}

To identify the common host factor working in both FMDV-IRES and CSFV-IRES, we have characterized the effect on Pycnogenol ${ }^{\circledR}$ (PYC) to FMDV-IRES activity, as PYC is natural product and reported to show anti-viral effect in some viruses. PYC was treated in cell lines B10 that express bicistronic dual luciferase mRNAs containing the FMDV IRES [14] for 72 hrs (Fig. 1). As shown in Fig. 1, FMDV-IRES activity was suppressed in a dose-dependent manner (Fig. 1A and no significant cytotoxicity (Fig. 1B) was apparent.

To identify the host factors affected by PYC treatment, we treated B10 cells with PYC and determined modifications in gene expression by microarray analysis (Fig. 2). We found 115 genes down regulated and 218 genes upregulated by more than 2 -fold following PYC treatment (Supplementary Fig. 1). We have selected 4 genes (No.1 4), polycystic kidney disease 1-like 3 (PKD1L3), ubiquitin specific peptidase 31 (USP31), signal-regulatory protein gamma (SIRPG) and hepatocyte growth factor (HGF) from the top 10 down regulated genes (Table 1-1) and 2 of the upregulated genes (No.5, 6), olfactory receptor family 6, subfamily N, member 1 (ORF6N1) (Table 1-2) for further analysis. We have chosen coding genes rather than non-coding genes and keratin which frequently affected by contamination during experiment.

\section{Characterization of host factors in FMDV-IRES-activity}

To further investigate the role of the candidate host factors (up- or down-regulated genes identified as described above), we targeted each selected gene with a specific siRNA in cell lines expressing the bicistronic reporter mRNA [9] (Fig. 3). Silencing of polycystic kidney disease 1-like 3 (PKD1L3), ubiquitin 
specific peptidase 31 (USP31) and signal-regulatory protein gamma (SIRPG) significantly suppressed FMDV-IRES activity (Fig. 3A) without cytotoxicity (Fig. 3B).

\section{Establishment of CSFV-IRES-expressing cell lines}

Whereas the FMDV-IRES is classified as a Type II picornavirus IRES (Fig. 4A), the CSFV-IRES is classified as an HCV-like IRES (Fig. 4B) [11]. To evaluate CSFV-IRES activity, we established cell lines incorporating a bicistronic reporter plasmid containing a CSFV-IRES [4] using a pCAGGS vector [9] (Fig. 5A). We further treated CSFV-IRES-expressing cells with PYC to ascertain any host factors possibly involved with the CSFV-IRES. Treatment with PYC suppressed CSFV-IRES activity in a dose-dependent manner without significant cytotoxicity (Fig. 5B).

\section{Role of host factors in IRES-mediated translation of CSFV}

To investigate host factors involved in this IRES, we targeted PKD1L3, USP31, and SIPRG with siRNA in CSFV-IRES-expressing cells (Fig. 6), because these host factors are also involved in FMDV-IRES activity (Fig. 3). Silencing of PKD1L3 and USP31 significantly suppressed CSFV-IRES activity (Fig. 6A) without cytotoxicity (Fig. 6B).

\section{Discussion}

In this study, suppressive effects of PYC on the activities of the FMDV-IRES and CSFV-IRES were observed. This may suggest the possibility of the antiviral effect of PYC for both FMDV and CSFV infection. We previously observed antiviral effect of PYC in HCV infection [2]. PYC suppressed HCV replication in subgenomic replicon cells and infection in HCV-JFH-1 [24] infected cells, and showed synergistic effect to interferon- $a$ and ribavirin, and was effective against viruses resistant to direct acting antiviral agents in vitro and in humanized chimeric mice [16]. PYC is a natural product, therefore, it may worth addressing the effect of PYC against FMDV and CSFV in future studies.

We identified host factors involved with the activity of both the FMDV-IRES and CSFV-IRES after ascertaining the suppressive effects of PYC on FMDV-IRES and CSFV-IRES activities. We found that PKD1L3 and USP31 may be common host factors that regulate the FMDV-IRES and CSFV-IRES elements. To the authors' knowledge, this is the first report linking PKD1L3 and USP31 with IRES function.

PKD1L3 was first identified as a candidate to regulate sour taste $[7,17]$. PKD1L3 cleaves the N-terminal G-protein-coupled receptor proteolytic site [10]. PKD1L3 is reported to form a complex with PKD2-L1 and regulating the influx-operated $\mathrm{Ca}^{2+}$ entry to produce $\mathrm{a} \mathrm{Ca}^{2+}$ spike in sensory responses [6]. USP31 is a deubiquitinating enzyme and activates nuclear factor-kappaB [23]. Variants in USP31 were strongly associated with adult-onset deafness in Border Collies [25]. The detailed role of PKD1L3 and USP31 in IRES activity should be addressed in a future study. 
In summary, the IRES-mediated translational activity of FMDV and CSFV may be a suitable target for the development of a wide range of antiviral drugs because of the relatively high sequence conservation of the IRES in each virus. Compounds that suppress PKD1L3 and USP31 could be developed as new antiFMDV and CSFV drugs, and the downregulation of PKD1L3 and USP31 could contribute to the establishment of FMDV and CSFV-resistant animal breeds.

\section{Abbreviations}

FMDV: foot-and-mouth disease virus; CSFV: classical swine fever virus; IRES: internal ribosomal entry site; 5'UTR: 5ç untranslated region; siRNA: short interfering RNA; PYC: pycnogenol; PKD1L3: polycystic kidney disease 1-like 3; USP31:ubiquitin specific peptidase 31

\section{Declarations}

\section{Acknowledgments}

The authors thank Professor Graham J. Belsham of the University of Copenhagen for his kind help and comments on the manuscript, and Mr. Henry Smith for his valuable comments on the English Edition.

Funding: This study was supported by a grant from the Ministry of Education, Science and Culture, Japan (grant number 20H3164).

\section{Compliance with ethical standards}

\section{Conflict of interest}

The authors declare that they have no conflict of interest.

\section{Ethical approval}

This study was performed in accordance with institutional committee protocols of Kagoshima University.

\section{References}

1. Burks JM, Zwieb C, Muller F, Wower IK, Wower J (2011) In silico analysis of IRES RNAs of foot-andmouth disease virus and related picornaviruses. Arch Virol 156:1737-1747

2. Ezzikouri S, Nishimura T, Kohara M, Benjelloun S, Kino Y, Inoue K, Matsumori A, Tsukiyama-Kohara K (2015) Inhibitory effects of Pycnogenol(R) on hepatitis C virus replication. Antiviral Res 113:93-102

3. Feng WY, Tanaka R, Inagaki Y, Saitoh Y, Chang MO, Amet T, Yamamoto N, Yamaoka S, Yoshinaka Y (2008) Pycnogenol, a procyanidin-rich extract from French maritime pine, inhibits intracellular replication of HIV-1 as well as its binding to host cells. Jpn J Infect Dis 61:279-285

4. Friis MB, Rasmussen TB, Belsham GJ (2012) Modulation of translation initiation efficiency in classical swine fever virus. J Virol 86:8681-8692 
5. Hashem Y, des Georges A, Dhote V, Langlois R, Liao HY, Grassucci RA, Pestova TV, Hellen CU, Frank J (2013) Hepatitis-C-virus-like internal ribosome entry sites displace elF3 to gain access to the 40S subunit. Nature 503:539-543

6. Hu M, Liu Y, Wu J, Liu X (2015) Influx-Operated Ca(2+) Entry via PKD2-L1 and PKD1-L3 Channels Facilitates Sensory Responses to Polymodal Transient Stimuli. Cell Rep 13:798-811

7. Ishimaru Y, Inada H, Kubota M, Zhuang H, Tominaga M, Matsunami H (2006) Transient receptor potential family members PKD1L3 and PKD2L1 form a candidate sour taste receptor. Proc Natl Acad of Sci USA 103:12569-12574

8. Jamal SM, Belsham GJ (2013) Foot-and-mouth disease: past, present and future. Vet Res 44:116

9. Kanda T, Ozawa M, Tsukiyama-Kohara K (2016) IRES-mediated translation of foot-and-mouth disease virus (FMDV) in cultured cells derived from FMDV-susceptible and -insusceptible animals. BMC Vet Res 12:66

10. Kashyap P, Ng C, Wang Z, Li B, Arif Pavel M, Martin H, Yu Y (2019) A PKD1L3 splice variant in taste buds is not cleaved at the $\mathrm{G}$ protein-coupled receptor proteolytic site. Biochem Biophys Res Commun 512:812-818

11. Khawaja A, Vopalensky V, Pospisek M (2015) Understanding the potential of hepatitis C virus internal ribosome entry site domains to modulate translation initiation via their structure and function. Wiley Interdiscip Rev RNA 6:211-224

12. Licursi M, Komatsu Y, Pongnopparat T, Hirasawa K (2012) Promotion of viral internal ribosomal entry site-mediated translation under amino acid starvation. J Gen Virol 93:951-962

13. Locker N, Easton LE, Lukavsky PJ (2007) HCV and CSFV IRES domain II mediate elF2 release during 80S ribosome assembly. The EMBO journal 26:795-805

14. Matsui T, Handa Y, Kanda T, Tsukiyama-Kohara K (2019) Silencing of the foot-and-mouth disease virus internal ribosomal entry site by targeting relatively conserved region among serotypes. Virus Genes 55:786-794

15. Matsumori A, Higuchi H, Shimada M (2007) French maritime pine bark extract inhibits viral replication and prevents development of viral myocarditis. J Card Fail 13:785-791

16. Mercer DF, Schiller DE, Elliott JF, Douglas DN, Hao C, Rinfret A, Addison WR, Fischer KP, Churchill TA, Lakey JR, Tyrrell DL, Kneteman NM (2001) Hepatitis C virus replication in mice with chimeric human livers. Nature medicine 7:927-933

17. Nelson TM, Lopezjimenez ND, Tessarollo L, Inoue M, Bachmanov AA, Sullivan SL (2010) Taste function in mice with a targeted mutation of the pkd113 gene. Chem Senses 35:565-577

18. Pacheco A, Reigadas S, Martinez-Salas E (2008) Riboproteomic analysis of polypeptides interacting with the internal ribosome-entry site element of foot-and-mouth disease viral RNA. Proteomics 8:4782-4790

19. Pacheco A, Martinez-Salas E (2010) Insights into the biology of IRES elements through riboproteomic approaches. J Biomed Biotechnol 2010:458927 
20. Reusken C, Dalebout TJ, Eerligh P, Bredenbeek PJ, Spaan WJM (2003) Analysis of hepatitis C virus/classical swine fever virus chimeric 5'NTRs: sequences within the hepatitis $C$ virus IRES are required for viral RNA replication. J Gen Virol 84:1761-1769

21. Tautz N, Meyers G, Thiel HJ (1998) Pathogenesis of mucosal disease, a deadly disease of cattle caused by a pestivirus. Clin Diagn Virol 10:121-127

22. Tsukiyama-Kohara K, lizuka N, Kohara $M$, Nomoto $A$ (1992) Internal ribosome entry site within hepatitis C virus RNA. J Virol 66:1476-1483

23. Tzimas C, Michailidou G, Arsenakis M, Kieff E, Mosialos G, Hatzivassiliou EG (2006) Human ubiquitin specific protease 31 is a deubiquitinating enzyme implicated in activation of nuclear factorkappaB. Cell Signal 18:83-92

24. Wakita T, Pietschmann T, Kato T, Date T, Miyamoto M, Zhao Z, Murthy K, Habermann A, Krausslich HG, Mizokami M, Bartenschlager R, Liang TJ (2005) Production of infectious hepatitis C virus in tissue culture from a cloned viral genome. Nature Med 11:791-796

25. Yokoyama JS, Lam ET, Ruhe AL, Erdman CA, Robertson KR, Webb AA, Williams DC, Chang ML, Hytonen MK, Lohi H, Hamilton SP, Neff MW (2012) Variation in genes related to cochlear biology is strongly associated with adult-onset deafness in border collies. PLoS Genet 8:e1002898

\section{Tables}

Due to technical limitations, table 1 and 2 is only available as a download in the Supplemental Files section.

\section{Figures}

\section{(A)}

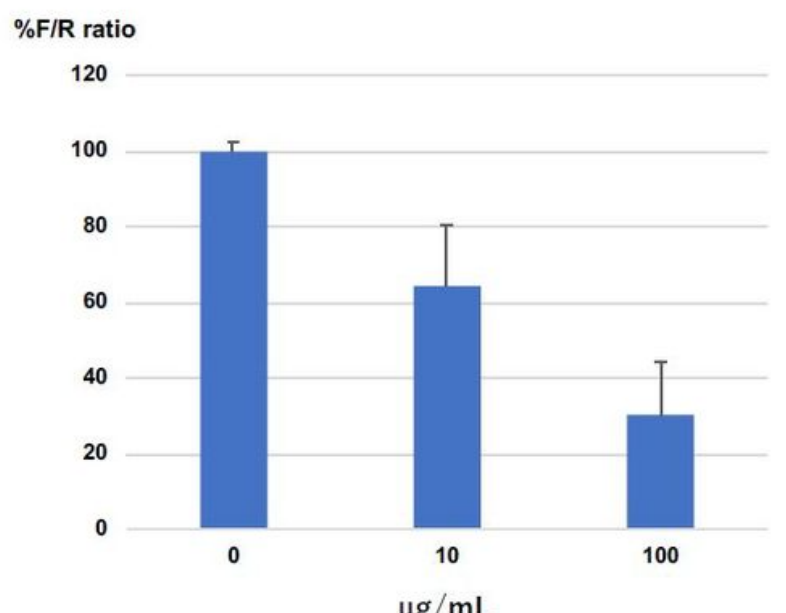

$\mu \mathrm{g} / \mathrm{mL}$

(B)

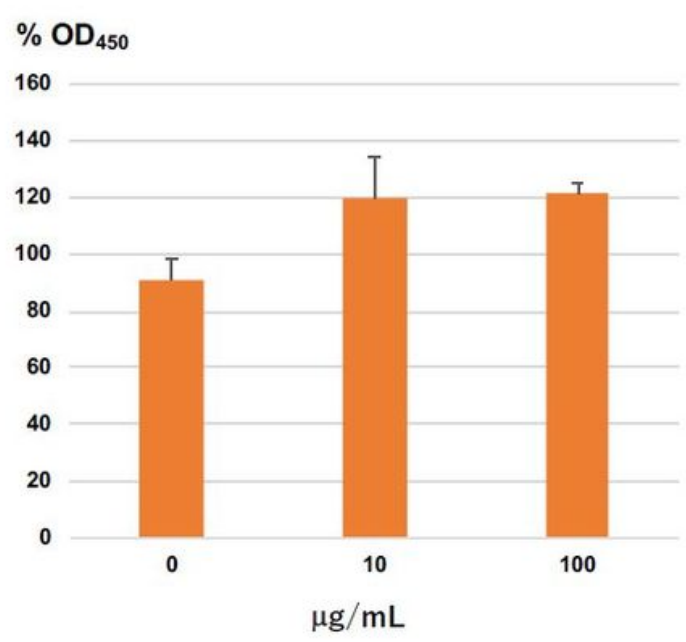

Figure 1 
Treatment of FMDV-IRES expressing cells with PYC. Mean values for triplicate samples are shown and vertical bars indicate S.D. FMDV-IRES expressing cell lines B5 (A) were treated with PYC at a final concentration of 0,10 and $100 \mathrm{gg} / \mathrm{mL}$. After $72 \mathrm{hrs}$ incubation, activity of Firefly and Renilla luciferase activities were measured, and IRES activity was calculated as the ratio of firefly luciferase activity to Renilla luciferase activity, plotted against the value for the untreated sample. (B) The ratio (\%) of the WST value (OD450) vs the untreated sample is shown.

\section{Scatter Plot(Processed Signal)}

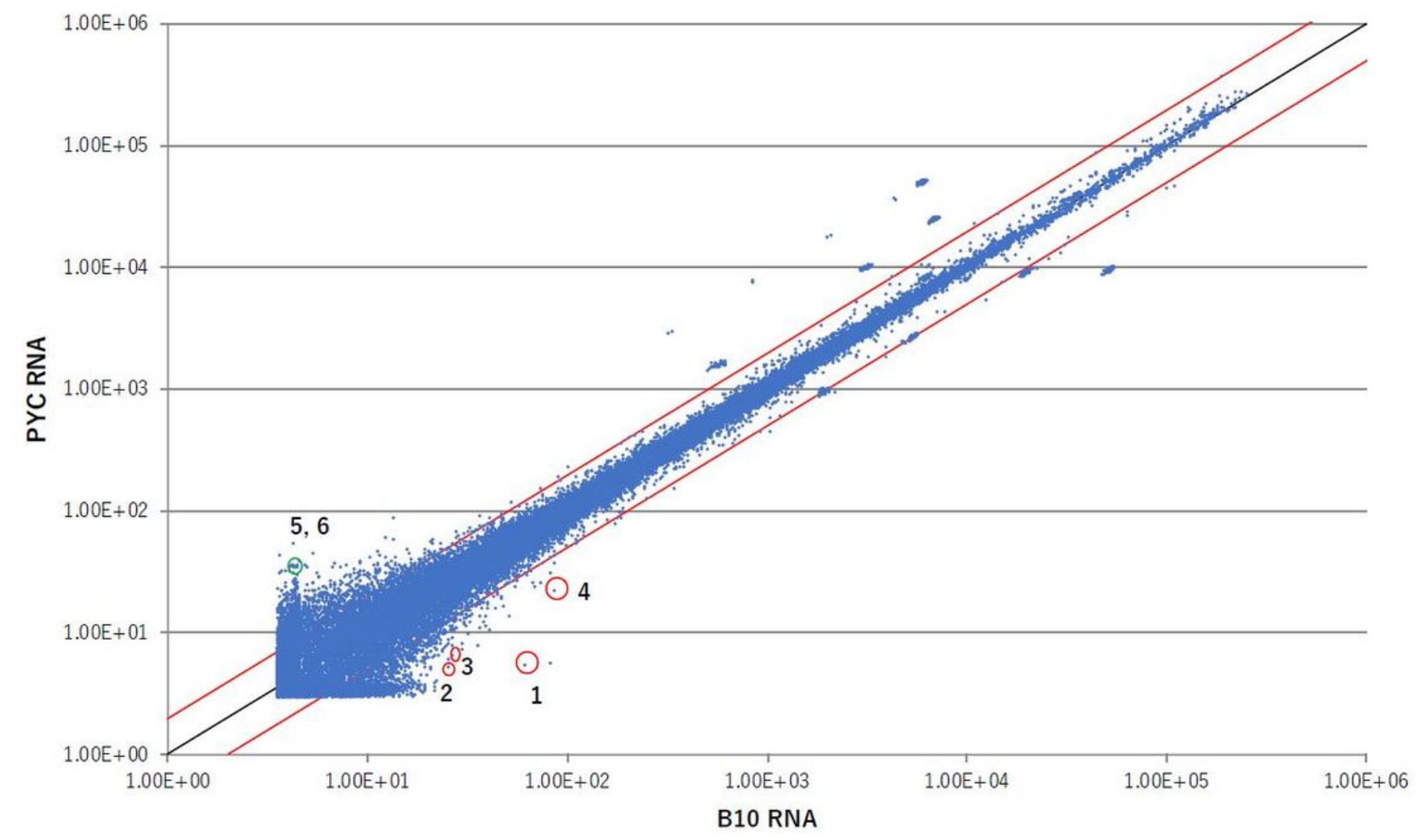

\section{Figure 2}

Result of microarray analysis are shown as a scatter plot. Cy3 labeled B10 RNA and cy5 labeled PYC treated B10 RNA (PYC) were characterized and spotted based on the gProcessed signal and rProcessed signal. Red lines indicate 2-fold change. Red circles numbered 1-4 indicate suppressed by PYC treatment and green circles numbered 5 and 6 indicate upregulated genes by PYC treatment, as summarized in Table-1. 


\section{$\%$ F/R ratio}

(A)

160

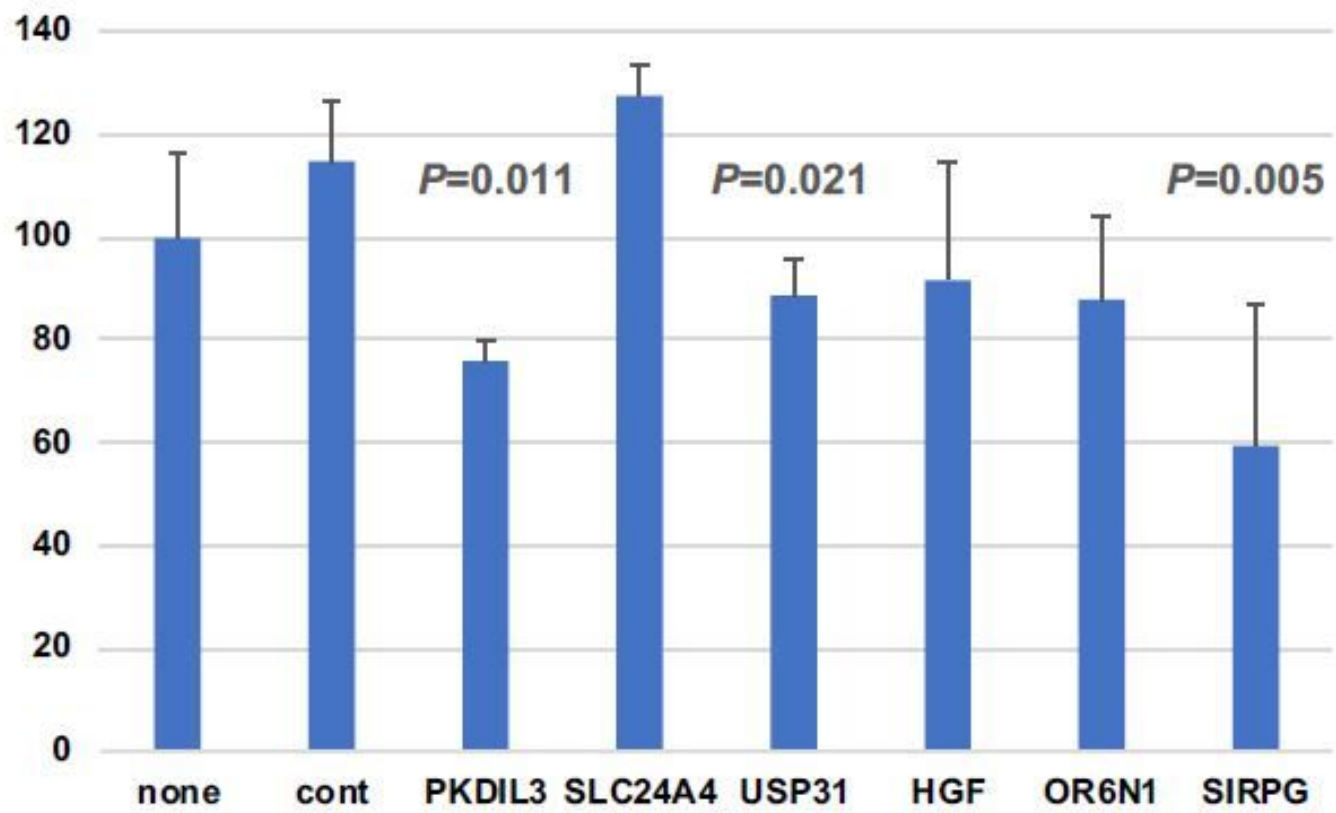

(B)

\section{$\% \mathrm{OD}_{450}$}

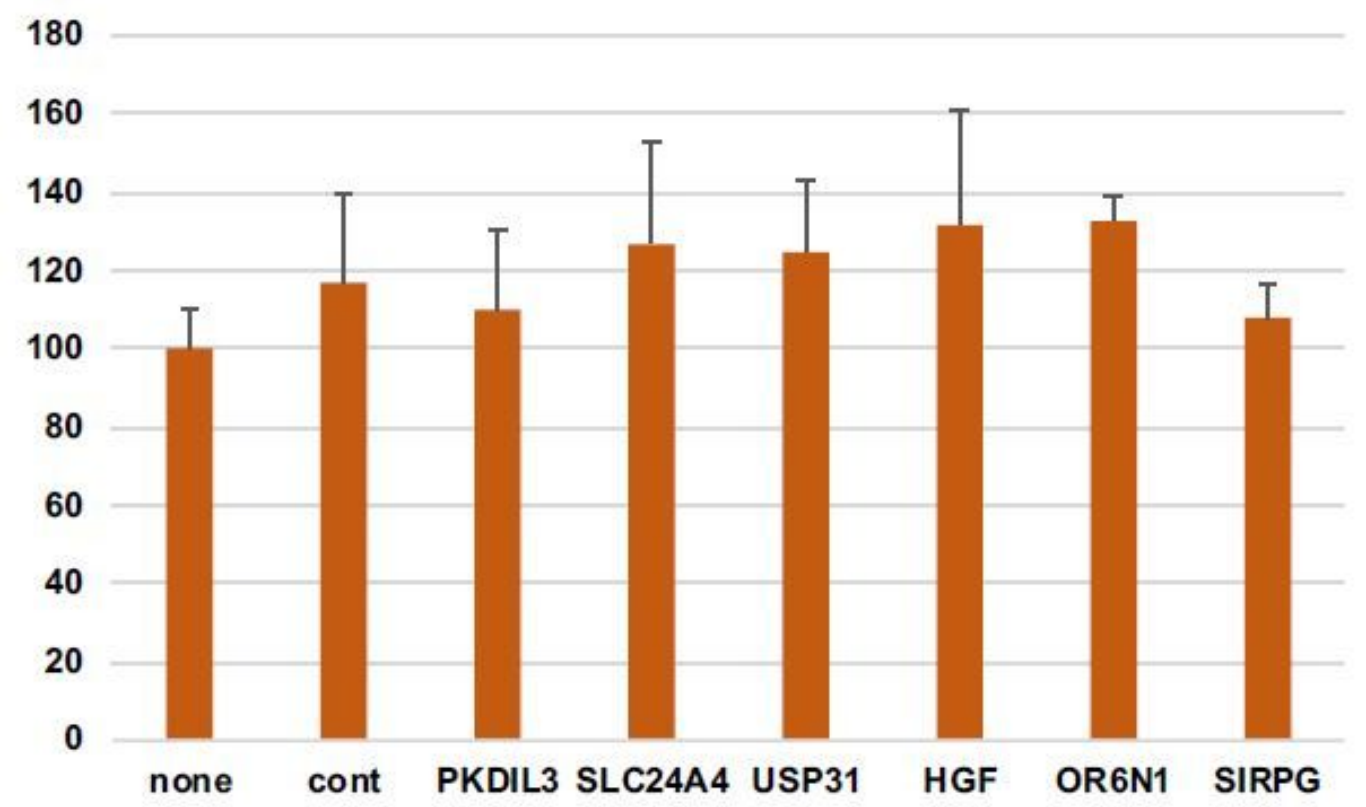

\section{Figure 3}

Treatment of siRNA of 6 factors in B10 cells. Mean values calculated for triplicate samples are shown, and vertical bars indicate SD. (A) IRES activity was calculated as shown in Fig.1. Statistical analysis was performed based on the student-t test comparing control siRNA treated cells and siRNA targeting 6 host factors. (B) The ratio (\%) of the WST value (OD450) vs. the untreated sample is shown. 

(A) Type II picornavirus IRES (FMDV-IRES)
(B) HCV-like IRES (CSFV-IRES)
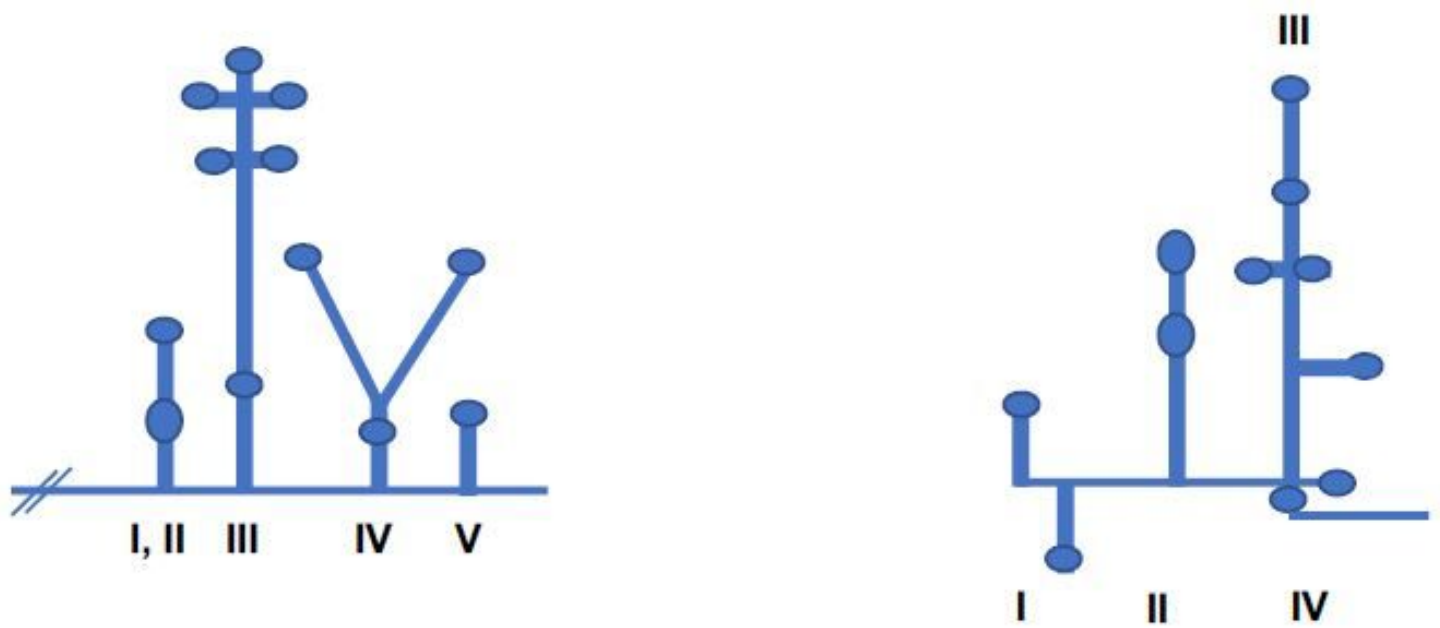

Figure 4

Secondary structure of FMDV-IRES and CSFV-IRES (A) Secondary structure of FMDV-IRES (Type II picornavirus IRES). Domain I V are indicated. (B) Secondary structure of CSFV-IRES (HCV-like IRES). Domain I IV are indicated. 


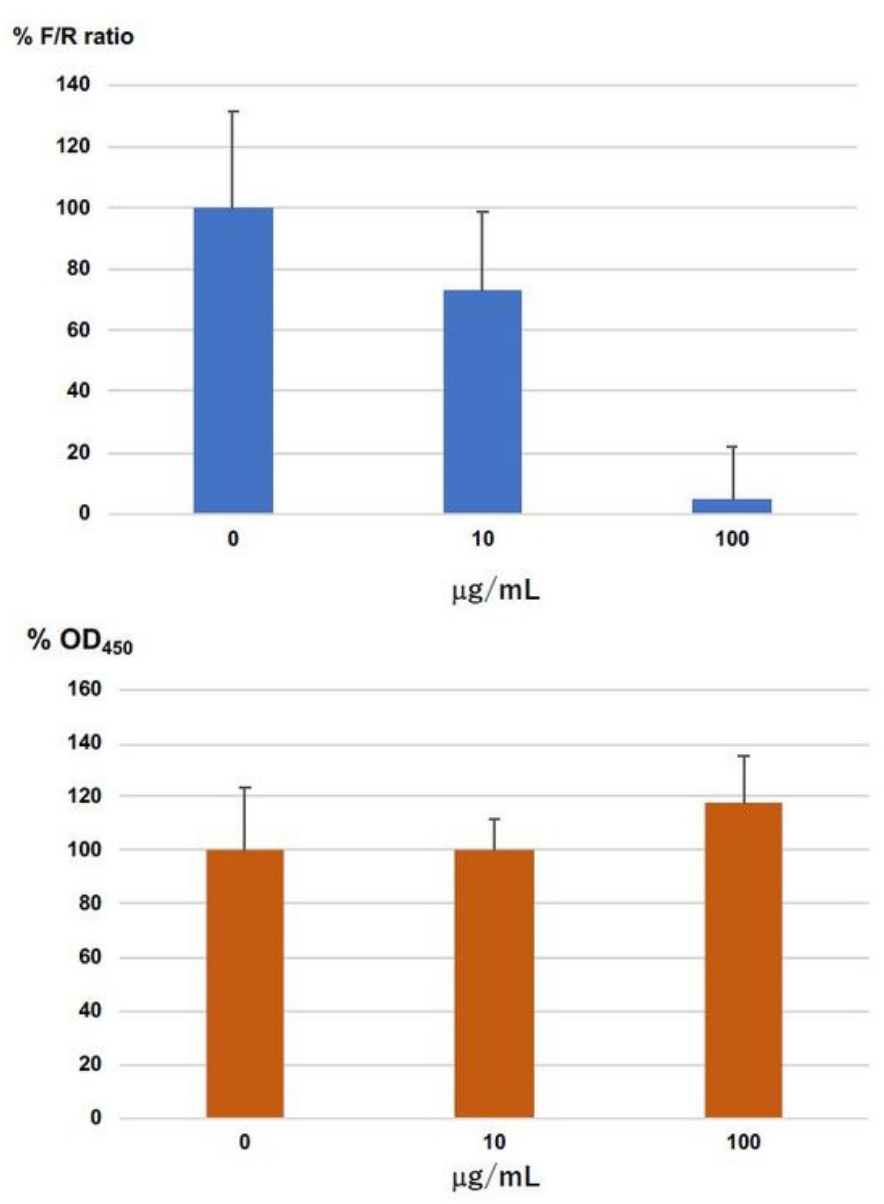

\section{Figure 5}

Structure of the bicistronic luciferase reporter construct and response to PYC (A) A bicistronic reporter construct was designed to contain the CSFV-IRES element located between the Renilla and firefly luciferase genes. The bicistronic reporter gene was cloned using the restriction enzymes EcoRV and BamHI and was ligated into the pCAGGS-Neo/MCS vector digested with EcoRV and BamHI. (B) CSFVIRES expressing cells were treated with PYC at a final concentration of 0,10 , or $100 \square \mathrm{g} / \mathrm{mL}$. After $72 \mathrm{hrs}$ incubation, activity of Firefly and Renilla luciferase activities were measured, and IRES activity was calculated as the ratio of firefly luciferase activity to Renilla luciferase activity plotted against the value for the untreated sample. The mean value for triplicate samples is indicated and vertical bars shows SD. The ratio (\%) of the WST value (OD450) vs. the untreated sample is shown in the lower column. 
(A)

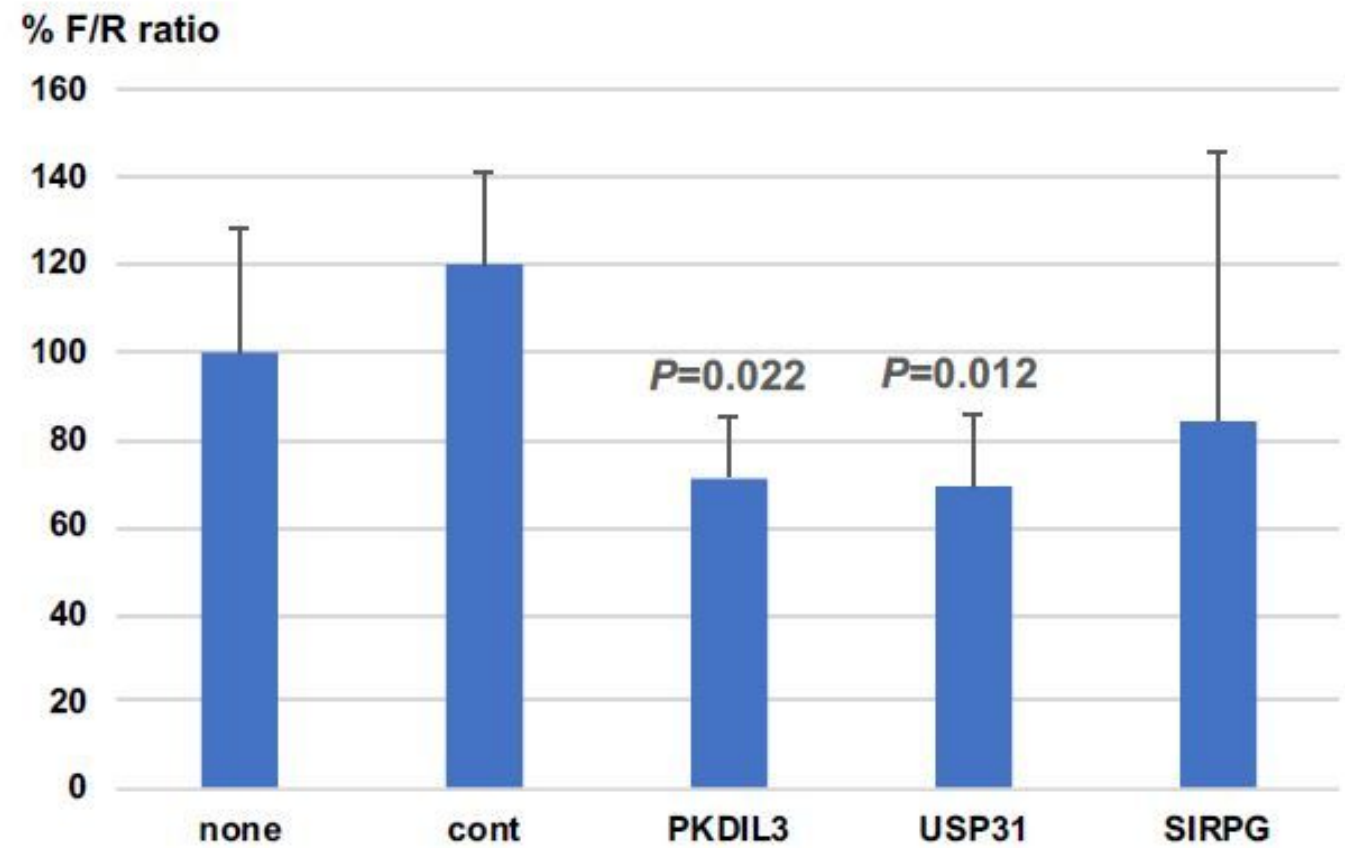

(B)

\section{$\% \mathrm{OD}_{450}$}

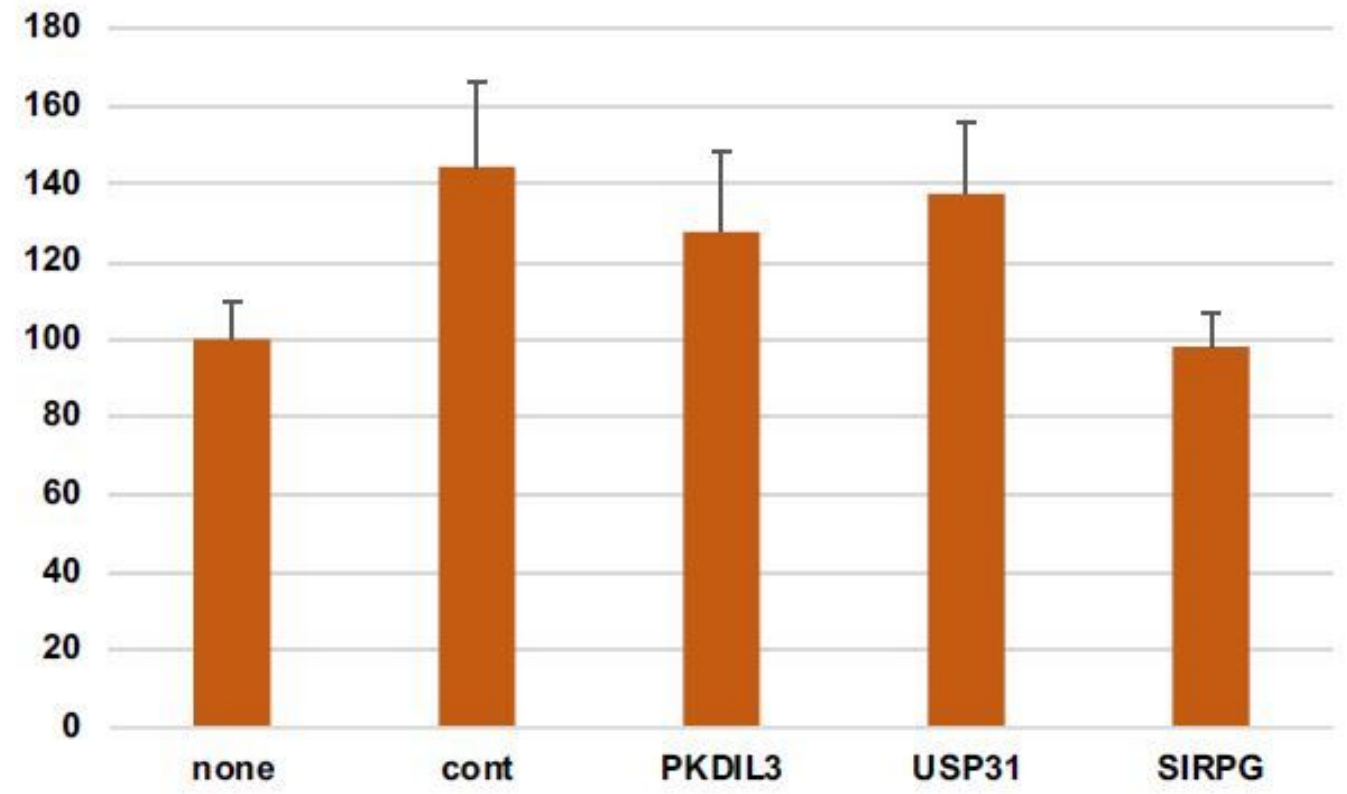

Figure 6

Effects of siRNA to PKD1L3, USP31 and SIRPG on CSFV-IRES activity siRNAs targeting PKD1L3, USP31 and SIRPG were reverse transfected into HEK293 cells (CSFV-IRES) using Lipofectamine RNAiMAX and incubated for $48 \mathrm{~h}$. Firefly (FMDV-IRES activity) and Renilla (cap-dependent translation) luciferase activities were measured. To evaluate IRES-mediated translational activity, the ratios of IRES-mediated translation vs. cap-dependent translation, and vs. untreated cells were calculated (A). Experiments were 
performed in triplicate, and error bars indicate standard deviations. Student's t-tests were performed to calculate $p$ values comparing control siRNA treated cells and cells transfected with PKD1L3, USP31 and SIRPG siRNA. (B) Cell viability was measured using WST assays by determination of the OD450 and ratios to the values for untreated cells were calculated. Experiments were performed in triplicate, and error bars indicate standard deviations.

\section{Supplementary Files}

This is a list of supplementary files associated with this preprint. Click to download.

- SupplementaryFig.1.xlsx

- Table1.xlsx 